\title{
Using Current Transformer and Three-phase Watt Hour Meter to Design of High Voltage Power Metering System
}

\author{
Dingzhen Li \\ Nanyang Institute of Technology \\ Henan Nanyang, China \\ e-mail: lidingzhenedu@163.com
}

\author{
Haizhen Guo \\ Henan Mechanical and Electrical Engineering College \\ Henan Xinxiang, China
}

\begin{abstract}
The high voltage power metering system is mainly composed of a current transformer (CT), voltage transformer (PT), consisting of two wire electric energy meter and connecting them. In the high-voltage power metering system, current transformer is important electrical equipment. Structure of current transformer is relatively simple, and it is consisting of mutually insulated winding, a two winding, iron core and frame, shell, terminal etc.. Threephase watt hour meter is used to measure the three-phase AC induction type meter active electric energy. The paper presents using current transformer and three-phase watt hour meter to design of high voltage power metering system. Simulation results show that this model can detect fault of current transformer.
\end{abstract}

Keywords-Current transformer; Three-phase watt hour meter; High voltage power metering; voltage transformer; Wire electric energy meter

\section{INTRODUCTION}

The high voltage power metering system comprises the electric energy meter, transformer and connecting them two wire three-phase active power; wiring mode of measurement used for three-phase three wire connection, connection so three phase active electric energy metering is more complex, the theory of 4071 kinds of wiring, and the right one, so measurement system failures are also many.

Due to a malfunction caused by normal connection of man-made or natural change measurement system, a long time measurement of engineering and technical personnel of electric power department according to this fault, mainly based on experience regular measure system. Detection means backward, mainly using current meter, voltage meter, phase meter measuring the corresponding voltage, current and phase values, and then draws a vector diagram to analyze and judge. This method of fault detection is more traditional, experience level dependent technical staff, and the process is complicated, so it is difficult to meet the real-time requirement.

In order to ensure the safe and economic operation of power system must be run on electric power equipment to monitor and measure. But the direct access measurement and protection device can not generally a high voltage equipment, need to be big current a system into small current scaling transformation, supply of measuring and protection device [1]. In the current measuring alternating current, for two instruments need to be converted into current unified (China two times rated current transformer for $5 \mathrm{~A}$ or $1 \mathrm{~A}$ ), voltage in addition lines are as high as the direct measurement is very dangerous.

The current transformer has variable flow and electrical isolation. It is the sensor in power system relay protection measuring instrument, such as the two devices to obtain information current of the first circuit, current transformer will be high current into the low current proportional, one side of the current mutual inductor connected in a system, the two sides is connected with the measuring instrument, relay protection.

The current transformer is used to measure the current relatively large. Current transformers takes the high current according to a certain proportion into a small current, provides current various kinds of instrument use and relay protection, and will be the two time the system isolation and high voltage. It is not only to ensure the safety of people and equipment, also making simple, meter and relay of standardization, improve economic benefit.

The high voltage power metering system is mainly composed of a current transformer (CT), voltage transformer (PT), consisting of two wire electric energy meter and connecting them. These components in a failure will cause the inaccurate measurement, or even lead to the paralysis of the whole high pressure measurement system. Current transformer short-circuit detection is a key technology of measurement; fault detection system at present, this technique is almost blank in practical application. How to detect the fault, especially a shortcircuit fault current transformer is particularly important. The paper presents using current transformer and threephase watt hour meter to design of high voltage power metering system.

\section{DEVELOPMENT OF High Voltage POWER METERING SYSTEM BASED ON CURRENT TRANSFORMER}

The main function of current transformer: one, the two equipment measuring instrument, relay and an isolating circuit, which can prevent a high voltage circuit of relay instrument, introduced two equipments, ensure the safety of the people; and also can prevent fault two instrument and the influence on the normal operation of a relay. Two, expand the scope of application of instrument, relay, the current a system into small current, are respectively used to provide current to the coil current measuring instrument, relay, correctly reflect the normal operating parameters and fault of electrical equipment. Three, to achieve 
standardization, miniaturization two side device, which has the advantages of simple structure, convenient installation specifications unified, screen, easy to maintain.

In addition, a side open circuit to make the two secondary side voltage of several hundred volts, once touch will result in electric shock accident. Therefore, the two side of the current transformer are equipped with a short circuit switch, prevent a side open circuit. In the use process, the two sides should be immediately removed once the open circuit load, and then, to stop processing. All the handle rear can use, in order to meet the needs of measuring instrument, relay protection, circuit breaker failure judgment and fault filtering device, in the generator, transformer, line, bulbar sectional breaker, bus breaker, bypass circuit breaker circuit are equipped with $2 \sim 8$ times two winding current transformer. For high current grounding system and it is according to the general threephase configuration; for small current grounding system, according to the specific requirements according to twophase or three-phase configuration.

Straight through current transformer itself structure without a winding, a current carrying conductor (load current) from L1 to L2 through the made of silicon steel sheet rolling roll round (or other shape) to a winding iron core [2]. The two winding uniformly wound around a circular core, in series with a two load current coil instrument, relay, transmitter, form a closed loop, as shown in Fig.1.

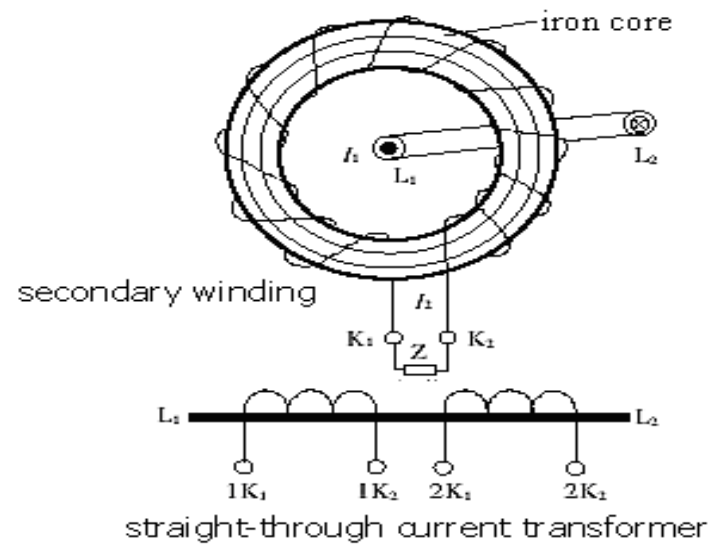

Figure 1. Development of High Voltage Power Metering System based on Current Transformer.

Two times the impedance circuit of current transformer connected instrument is small, the running state is equivalent to transformer short-circuit state, the core section is larger in the design and manufacture, in order to reduce the magnetic flux density and the excitation current to improve accuracy, therefore, that can approximate, the first winding ampere turns is equal to two times winding ampere turns.

The current transformer of the type, and it is a winding unchanged, in the winding two windings, add a few taps, to obtain a plurality of different ratio. It has a core and a winding turns a fixed number of secondary windings, insulation copper wire is wound on the insulating cylinder is sheathed on the core of the two winding tap, different ratio of extraction, connected to the connection terminal seat, each tap set terminals respectively, thus forming a plurality of ratio.
Current transformer in use if occurred two times open circuit, the two winding F2 is equal to zero, a winding MMF F1 remains unchanged, and all for the excitation magnetic potential $\mathrm{F} 0=\mathrm{F} 1$, synthesis, synthesis of magnetic potential when $\mathrm{F} 0$ is lower than that of the normal time (F1-F2) increased many times, so that the magnetic core the dramatically increased and reached saturation. Because of core saturation causes the flux waveform into flat wave, because the change of induced electromotive force is proportional to the flux rate of $\mathrm{D} \varphi / \mathrm{dt}$, so this is the two time in the winding will induce EMF E2 high. The two winding open circuit when E2 induction electromotive force two winding is non sinusoidal wave peaked, the peak of up to thousands of volts of high.

The working principle of current transformer by current transformer equivalent circuit in Figure 1 to illustrate, as with the voltage transformer, equivalent circuit of current transformer could be more intuitive painting into two independent loops, but for the convenience of analysis, the method is similar to the voltage transformer, the transform " $\mathrm{T}$ " type of equivalent circuit [3]. On the current grid primary winding, the induced EMF is in the two secondary winding, resulting in two current. Because the two winding transformer in the resistance and reactance of memory components, connected to the two loop of the electric energy meter and other instruments are resistance and reactance component. Here, a reactance is sensitive, transformer ratio is equation (1).

$$
E\left\{\bar{v}(m, s)\left(\tilde{\gamma}^{(\eta)}(m, s-1)\right)^{T}\right\}=0
$$

Where $\mathrm{E}$ is the electric energy meter, $\mathrm{m}$ is the reactance component, $\mathrm{s}$ is the memory components. In the same load conditions, in order to ensure the energy measurement accuracy requirements become smaller, some (to meet the load current in a rating of $2 / 3$ or so), accuracy is higher (such as 1K1, 1K2 200/5, 0.2); and the relay protection of electrical equipment, taking into account the protection factor of fault the current large, requires becomes larger, accuracy can be slightly lower (such as $2 \mathrm{~K} 1,2 \mathrm{~K} 2300 / 5,1$ ).

A secondary winding is adjustable, two multi winding current transformer. The current transformer is the ratio range, but also can change, in the high voltage current transformer. The first winding is divided into two sections, respectively, through the iron core transformer, two winding is divided into two independent windings are tapped, different level of accuracy.

The main parameters of the current transformer, accuracy class, according to JJG313-1994 "measuring current transformer verification regulation", but the accuracy of current transformer is divided into: 0.001, $0.002,0.005,0.01,0.02,0.05,0.1,0.2,0.5,1$. Error of transformer includes ratio difference and phase difference, transformer each accurate grade have clear requirements.

Combined transformer by current and voltage transformer assembly, is installed on the high voltage metering box, cabinet, used for measuring electric energy or power function of electric equipment of relay protection device. Combined current and voltage transformer is two or three sets of the current transformer primary, two secondary winding and iron core and the voltage 
transformer secondary windings and iron core one or two, fixed on the steel body structure, body immersed with transformer oil, the one or two winding are connected with high voltage porcelain, lead, in the box body, forming an integral insulating, closed, as is shown by equation (2).

$$
\hat{X}_{i}(k \mid k-1)=F(k-1) \hat{X}_{i}(k-1 \mid k-1)
$$

Ratio of current transformer size if the choice is undeserved can cause the error changes affect the correct measurement. "Regulation device technical measurement management power" (DL/T448-2000)) states: "to determine the current transformer rated primary current, should ensure the normal operation of the actual load current reaches the rated value, at least not less than." We are in actual use, changes in the scope of current should be in, because the current transformer excitation current, the proportion of small, relatively accurate measurement.

Current transformer current through the coil two sides reflects the current of the first circuit corresponding phase. The connection is usually measured current in the load balanced three-phase circuit, or in relay protection for overload protection wiring, as is shown by equation(3) [4].

$$
P V(x)=\frac{1}{y_{2}-y_{1}} \sum_{y=y_{1}}^{y_{2}} I(x, y), \quad x \in\left(x_{1}, x_{2}\right)
$$

Structure of current transformer is relatively simple, consisting of mutually insulated winding, a two winding, iron core and frame, shell, terminal etc.. The working principle and the transformer is basically the same, a number of turns of the secondary winding (N1) less, direct series to the power supply circuit, a load current () through a winding, alternating magnetic induction produced by the two current in proportion to reduce (two); the number of turns of the secondary winding (N2) more the two time, and load current coil instrument, relay, transmitter, $(\mathrm{Z})$ closed loop forming series, as is shown by Fig .2.

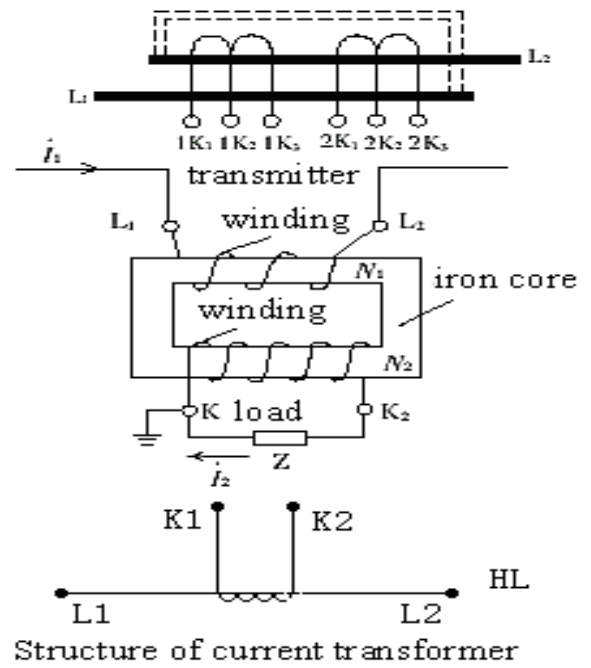

Figure 2. Structure of current transformer.

Two phase $\mathrm{V}$ connection is also called phase relay. Two current coil devices current is equal to two times the current transformer, it reflects a phase current. According to the current public line, side two transformers, is just the inverse missed transformer B phase two current, so the three current coil of the wiring reflect respectively, threephase current, widely used in neutral non grounding and load balance and not regardless of three-phase three wire circuit, for the relay protection and measurement.

The two winding tapped itself is divided into two different ratio and different level of accuracy of winding, with a winding connection piece position changes, a winding number changed, the ratio is changed, so that the formation of multiple range ratio, different ratio of two independent winding tapped and different grades of accuracy, can be respectively applied to electric energy metering, indicating instrument, transducer, relay protection, in order to satisfy different use requirements.

Current transformer selection is too large, i.e. when the excitation current working at rated current, because the inner core saturation excitation current is large, negative error is very serious, and thus less of electric energy [5]. If the long-term overload operation, it will burn out winding or shorten the service life of the current transformer, serious when may burn the current transformer. Current transformer ratio selection is too small, i.e. when the excitation current is below the rated current, will reduce the accuracy, but also can have the negative error.

In recent years, many technical personnel has carried on the analysis to the two circuit of current transformer in one phase short circuit or phase to phase short circuit, voltage transformer secondary circuit two one phase open circuit, current transformer polarity reversal caused by inaccurate measurement problems [6]. These studies have a common characteristic, namely the known metering system has some sort of malfunction, the comparison under normal condition and fault condition to solve the loss of value, the value of electricity. However, the practical application is required for real-time measurement system itself or the type of external causes of failure, and can real-time calculate loss measurement.

\section{RESEARCH OF THREE-PHASE WATT HOUR METER WITH CURRENT TRANSFORMER}

The load current is large scale measurement of threephase active power, except you can use the rated current of three phase active power larger energy meter, can also be a table with current transformer of three-phase active power With the current transformer, because the two current transformers is $5 \mathrm{~A}$, the rated current of electric energy meter should also use the $5 \mathrm{~A}$, the relation is called electric energy meter match the current transformer.

DS series of electric energy meter for three-phase three wires (three phase two element); DT series of electric energy meter for three-phase four wires (three phase three element). The three-phase load balance with three-phase three wire electric energy meter; unbalanced three phase four wire electric energy meter. Rated current and voltage according to the actual load and the mode of connection and. Electric energy meter rotating part friction is large, light load due to rotation torque is small, a larger error will be produced. To make regular load close to the electric energy meter rated current, to minimize the error, as is shown by equation (4). 
$L\left(x_{1}, x_{2}, \cdots, x_{n} ; \hat{\theta}\right)=\max _{\theta \in \Theta} L\left(x_{1}, x_{2}, \cdots, x_{n} ; \theta\right)$

High voltage power metering needs the high current high voltage into a small current low voltage measurement by current and voltage transformer, so under normal circumstances of current transformer is almost in a shortcircuit state, the magnetization force a current to produce the most current is compensated by two times, the total magnetic flux density is small, the two winding potential is not. When the open circuit current transformer secondary circuit two impedance, infinite, current equal to zero, a current into the excitation current, the potential is generated very high in the two winding, a threat to personal safety, causing the appearance, protection device, transformer insulation damage two times [7]. The two side of the current transformer one phase short circuit, connected to the phase of the electric energy meter will stop; the two phase to phase short circuit, electric energy meter can not measure the user correct electricity use for it.

For three-phase four wire connection, there are generally two kinds of the transformer access measurement methods: direct access to the voltage, current CT access (complete star connection), and this method is mainly used for $380 \mathrm{~V}$ three-phase AC users of low voltage electric energy metering, as shown in Fig .3 [8]. Voltage by the PT access (complete star connection), and it is current through CT access type (complete star connection), this method commonly used in the above voltage electric energy metering.

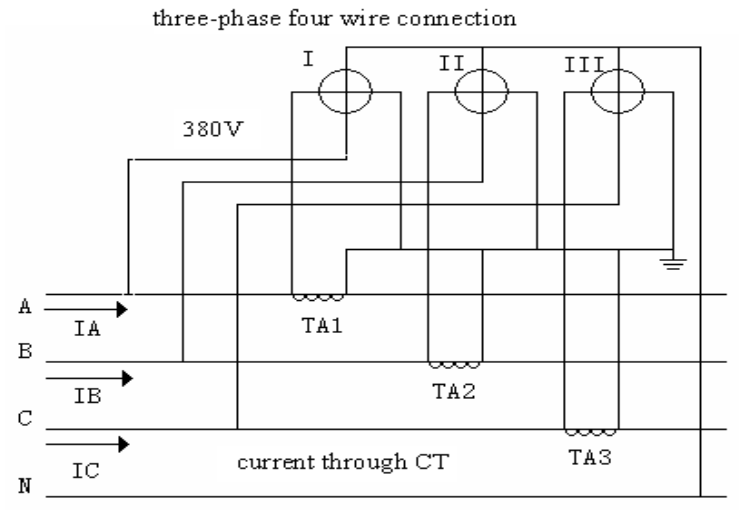

Figure 3. The three-phase four wire CT access mode diagram.

The current short circuit and voltage disconnect method: A, B, C short circuit current import line or disconnect from the $\mathrm{A}, \mathrm{B}, \mathrm{C}$ phase voltage, see electric energy meter rotary speed, if the load is more stable and balanced, short with a phase current or disconnecting a phase voltage, power table speed to normal $2 / 3$, if the deviation is big, show abnormal measurement. Also available at the same time to send the same phase voltage, current approaches are examined, the electric energy meter speed should be as normal 1/3. Terminal box voltage calibrator from electric energy meter, current probe current transformer secondary side clamp from two current transformer in sampling, check to observe the relevant parameter apparatus on the screen (such as with a clip on transformer calibrator can be easily measured ratio error of current transformer), is helpful to differentiate wiring, current, voltage is normal, if the power meter error to be removed to check.

The table is used to measure the three-phase AC induction type meter active power of three-phase active power, can be divided into two phase and three-phase three element, two element.

Three-phase active power short circuit of the primary side of current transformer measurement system, electric energy meter current coil short circuit of a certain phase or interphone short circuit; voltage coil of an electric energy meter of one phase or two-phase disconnect. These all cause the meter can not correctly measure the power users of electricity. In the high-voltage power metering system, current transformer is important electrical equipment, contact element between a system and the two systems, and it is widely applied in the analysis of relay protection, system monitoring and system.

\section{USING CURRENT TRANSFORMER AND THREE-}

\section{PHASE W ATt Hour METER TO DESIGN OF High Voltage POWER METERING SYSTEM}

In power system, transmission, process, electrical energy measurement especially for high voltage and large capacity electric energy measurement is very important, because it is not only related to the safe and economic operation of the power production and direct economic benefits, but also directly, indirectly linked to the beneficial to the people's livelihood and economic benefit social benefits. Therefore, a set of comprehensive and accurate electrical energy measurement is an important part of electric metering of electric power system.

High voltage power supply high voltage metering (referred to as high) is the national standard voltage 10KV widely used in rural and urban areas and high voltage power supply system more (internal power supply system of China's large industrial and mining enterprises generally use the $6 \mathrm{KV}$ high voltage power supply). The power supply for high voltage, high current, thus measuring battery voltage and current respectively through the voltage transformer (PT) and current transformer (CT), and in the two secondary side voltage transformer and current transformer connected energy meter to measure. The measurement of the electrical energy meter rated voltage: (three-phase three wire element (two) or threephase four wire three element); rated current: 1 (2) A, 1.5 (6) A, 3 (6) A.

The high voltage power metering system comprises the electric energy meter, current transformer, voltage transformer (CT) (PT) and two wires. Any part of them failure or due to natural or man-made wiring errors can lead to paralysis can not correctly measure even the whole measurement system, so that the failure mode it with wiring and parts of the state.

Because of a short circuit fault is man-made (electricity). While the two circuit may be naturally occurring may also be man-made, no matter what the reason is the electric energy meter can not be measured correctly the user consumption, so find a way to detect the fault, is of great academic and practical significance [9]. Therefore, to establish a real-time fault type metering system model is essential for high voltage power metering system of modern detection. Based on this idea, the short circuit of the primary, the two side of the current 
transformer for example, try to change the circuit parameters to establish the model of fault detection.

Through the analysis of the calculation according to the detection signal changes were obtained under network voltage can not determine whether there was a side of the current transformer short-circuit fault, because according to the detection signal size change is not judged to be due to the change of load or due to a fault current transformer, a short circuit caused by. Therefore need to add the last frequency in the detection circuit standard voltage signal is different from the power frequency signal and a standard resistor. Superposition of signal detection is the detection signal is obtained when the detection signal and the standard voltage source effect under the action of the power grid.

The simulation is based on a short circuit fault current transformer was studied as an example, the two short circuit current transformer has a similar result with a short circuit fault, which can be used to analyze network impedance calculation, this two kinds of fault, the network impedance have changed dramatically, and this change is not possible because the load variation, so it can happen to judge the fault according to the change of signal.

This paper analyzes the network faults on high-voltage power metering system; secondly, to establish the current transformer one or two short circuit fault detection model; finally, so is the computer simulation to verify the effectiveness of the fault detection model based on CT a short circuit as an example, and explains the model for detection of fault current transformer short circuit two times as effective.

In many high voltage power metering system of electric energy meter wiring error, error measurement circuit wiring way according to the access mode of electric energy meter and electric energy meter type (single-phase, three-phase three wire, three-phase four wire type) and different. Such as direct access single-phase electric energy meter wiring error probability is 15 ; the single phase electric current transformer access energy meter wiring error probability 20; direct access to three phase three wire electric energy meter wiring error probability is 709 , the three-phase current transformer access three wire electric energy meter wiring error probability 750, a three-phase access voltage transformer and current transformer three wire electric energy meter wiring error probability is 4071 ; direct access or a three-phase transformer access four wire electric energy meter wiring errors in it, is as shown in Fig .4.

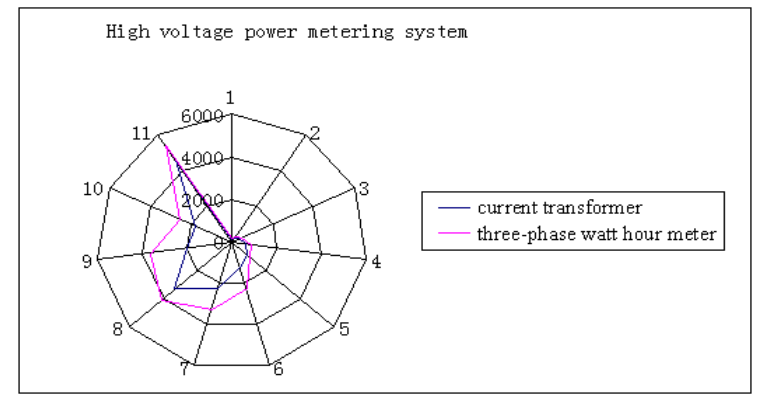

Figure 4. Design high voltage power metering system based on current transformer with three-phase watt hour meter.
As long as one side of the current transformer is not short, this value is not likely to close to 0 . When a current transformer short circuit, and the impedance is close to 0 . From Fig. 4 it is clear that, along with the load changes, changes in the scope of detection signal is approximately, and the current transformer has a primary side short circuit when the voltage is almost 0 , if the voltage detection signal suddenly becomes zero, is not likely to be due to changes in the load, can judge when a current transformer (CT) the short circuit of the primary side.

\section{SUMMARY}

The paper presents using current transformer and threephase watt hour meter to design of high voltage power metering system. Form the two wire high voltage power metering system is composed of a current transformer, a three-phase electric energy meter and connecting them, any one of them will result in the failure of electricity metering system can not measure the user right. Because of this, the high voltage power metering system fault and detection method becomes a hot issue of research, especially the problem of detecting current transformer short circuit fault. Analysis of the current transformer for high voltage measurement system, a two short circuit fault, according to the causes of fault, a fault detection model, gives the detection circuit, the simulation results show that the model checking of the fault is very effective, so as to solve the problem of current type of electrical detection.

\section{REFERENCES}

[1] Tanini, moh Tamimi, Mohammad, "Short Term Electric load forecasting Via fuzzy neural collaboration", Electric Power Systems Research, 2000.56(3):243-248.

[2] Han Song-lin, Tang Ling, "The influence on the measurement of wrong circuit of the electric meter", Electrical Measurement and instrumentation, 1999, 36(2):15-19.

[3] Han Song-lin, "Research of the functions of guanding against the theft of electricity", Electrical Measurement and instrumentation, 1999, 36(7):8-12.

[4] Yuzhong Li, "Research and Design on a Novel Optoelectronic Current Transformer Based on FPGA and DSP", JDCTA, Vol. 6 , No. 7, pp. $206 \sim 215,2012$.

[5] Kim, Kwang-Ho; Youm, Hyoung-Sun; Kang, Yong-Cheol, "Shorttime forecasing for special days in anomalous load conditions using neural networks and fuzzy inference method", IEEE Transactions on Power System, 2000 15(2):559-565.

[6] Liang, R-H, Cheng, C.-C, "Short-term load forecasting by a neurofuzzy based approach, International journal of Electrical Power and Energy System”, 2002 .24(2): 103-111.

[7] Tai-Ying Zheng, Yong-Cheol Kang, "Development of a compensating algorithm for an iron-cored measurement CT using flux-magnetizing current curves And voltage-core loss current curves", RNIS, Volume 4, pp. 12 18, 2009.

[8] LUO Yan, Duan Xiongying, ZHANG Minzhi, DONG Enyuan, "Research on the Electronic Transformer Applied in IEC 61850-92 Process Bus", IJACT, Vol. 4, No. 13, pp. $217 \sim 224,2012$.

[9] Heon Gyu Lee, Yonghoon Choi, Jin-ho Shin, "Spatio-Temporal Mining for Power Load Forecasting in GIS-AMR Load Analysis Model", IJIPM, Vol. 2, No. 1, pp. 57 66, 2011 